\title{
Comparative Drug Utilization of Antimicrobial Agents in Medical and Respiratory Intensive Care Units of a Tertiary Care Teaching Hospital in South India
}

\author{
Bincy Benjamin', B Sajeev Kumar1, Padmaja Udaykumar², V.B. Narayana Swamy1 \\ ${ }^{1}$ Department of Pharmacy Practice, Karavali College of Pharmacy, Mangalore-575 028, INDIA. \\ ${ }^{2}$ Department of Pharmacology, Father Muller Medical College Hospital, Mangalore-575 002,INDIA.
}

\begin{abstract}
Introduction : Drug Utilization Evaluation play a key role in managing healthcare system to understand, interpret, evaluate and improve the prescribing, administration and use of medications. Objective: To evaluate and compare the drug utilization of antimicrobials in Medical and Respiratory Intensive Care Units so as to include restriction policies. Methods: A prospective, observational study was performed on 209 prescriptions. The total number of drugs, dose, route, sensitivity pattern and cost were collected from in-patient records. The Defined Daily Dose/100 bed days of the most frequently prescribed antimicrobials was also calculated. Results: Out of the 1046 drugs prescribed in the Medical Intensive Care Unit, 167 antimicrobials were prescribed with an average of 2 drugs per prescription. The average length of stay was $4( \pm 1.4)$ days. Frequently prescribed antimicrobials were ceftriaxone, amikacin and clindamycin. The DDD/100 bed days for those drugs was 22.2, 6.9 and 5.9 respectively. The widely prescribed combinations were piperacillin + tazobactam and cefoperazone + tazobactam. In only $23 \%$ of the cases sensitivity test was performed. The most prevailing organisms were E.Coli (53\%) and Acinetobacter sps (23\%). Colistin and meropenam were the most sensitive antimicrobials for most microorganisms. In Respiratory Intensive Care Unit, out of 1024 drugs, 163 antimicrobials were prescribed with an average of 2 drugs per prescription. The average length of stay was $4( \pm 1.4)$ days. Ceftriaxone $(40 \%)$ was found to be most commonly prescribed drug followed by clindamycin (17\%), levofloxacin (12\%) and meropenam (11\%). The generally prescribed combination was piperacillin + tazobactam (30\%). The DDD/100 bed days was found to be high. In only $20 \%$ of the cases sensitivity test was performed. Acinetobacter sps was the most frequently isolated pathogen and all microorganisms were sensitive to colistin. Conclusion: There is a need of anti-microbial agents usage guidelines and restriction policies for the rational prescribing of antimicrobials in critically ill patients.
\end{abstract}

Key words: Drug Use Evaluation, Antimicrobial Agents, MICU, RICU, Sensitivity pattern, Defined Daily Dose.

\section{INTRODUCTION}

Drug therapy plays a crucial role in improving human health by enhancing the quality of life and extending the life expectancy. A method to evaluate and improve drug use is by conducting Drug Utilization Studies (DUS). Drug Utilization is defined by the WHO as the marketing, distribution, prescription, and use of drugs in society with special emphasis on the resulting medical, social, and economic consequences. ${ }^{1}$ The World Medicines Situation Report of 2011 concludes that inappropriate antibiotic use, including overuse and misuse, is a serious global problem. Also, established and newly emerging infectious diseases are increasingly threatening the health of population. ${ }^{2}$

Intensive Care Unit (ICU) patients are a diverse group, who often suffer from severe illness, multiple organ dysfunction

DOI: 10.5530/ijopp.9.2.11

\section{Address for}

correspondence:

Dr. B. Sajeev Kumar,

Professor and Head-Pharm D

Programme, Department of

Pharmacy Practice,

Karavali College of Pharmacy,

$\mathrm{NH} .13$, Vamanjoor,

Mangalore -575028

D.K. Dist., Karnataka State, INDIA.

Phone no: +919495817507 ,

Fax: +910824226365

E-mail: drsajeev2016@gmail.com, bsajeev2001@yahoo.com

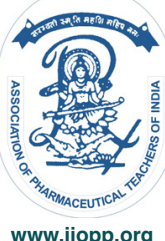

www.ijopp.org 
and concomitant diseases. Selection of drugs and initiating a drug therapy in critical care patients is a complex task and is usually difficult. Generally, antimicrobial agents (AMA) are the commonly prescribed drugs in an ICU setting. In such circumstances, optimal antibiotic use is crucial in the critical care setting, especially in an era of rising antibiotic resistance and lack of new antimicrobial development. ${ }^{3}$ In order to improve antimicrobial use, its outcome need to be measured. This is possible by introducing a proper guideline and monitoring process for the use of AMA. Such practices are highly beneficial as it can ensure rational drug therapy.

AMA measurement allows comparison, which can highlight differences in approach that might reveal opportunities for improvement, but can also provide assessment of risk, which might permit mitigation. ${ }^{4}$ Furthermore, calculating the cost of AMAs prescribed among patients admitted to ICU might be mandatory for future policies and procedures regarding antibiotic use in ICUs. ${ }^{5}$ The present study is aimed to explore and describe the current pattern of antimicrobials prescribing practices and utilization in critically ill patients.

\section{MATERIALS AND METHODS}

The study was conducted in Medical and Respiratory Intensive Care Units (MICU and RICU) of 1300 bedded medical college \& teaching hospital. A prospective hospital based non-experimental (observational) study was carried out for a period of 6 months. Ethical clearance was obtained from the Institutional Ethics Committee before starting the study. A total of 209 subjects were included in the study (MICU-110, RICU-99). The inclusion criteria was patients above 15 years and who were admitted in MICU and RICU exclusively. Patients with incomplete data, and treatment charts without AMAs were excluded from the study.

The data source needed for the study was collected from case reports, treatment charts and lab reports in a specially designed patient data entry form. The cost details of AMAs were collected from pharmacy and hospital formulary. The outcome measurement was measured using the below mentioned formulae. ${ }^{6}$

DDD / 100 bed days $=\frac{\begin{array}{l}\text { No. of units administered } \\ \text { in the study period }(\mathrm{g}) \times 100 \\ \mathrm{DDD}(\mathrm{g}) \times \text { No. of days in the study period } \times\end{array}}{\text { No. of beds } \times \text { Occupancy index }}$

where,

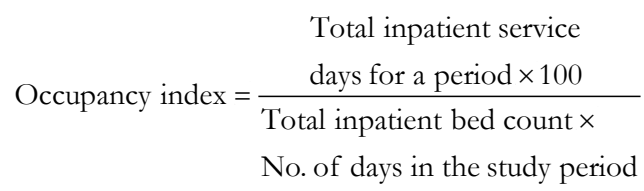

The average cost of most commonly used AMA and AMA combinations were calculated by multiplying cost per unit dosage and the number of dose used in each patient.

\section{Statistical Analysis}

Data were entered into Microsoft Excel (Windows 7; Version 2007) and analyses were performed accordingly. Descriptive statistics such as frequencies and percentages were calculated for categorical variables. Mean $( \pm)$ and standard deviation $( \pm)$ were computed for continuous variables. Graphic representations were used for visual interpretation of the analyzed data.

\section{RESULTS}

\section{Demographic Profile and Patient Characteristics}

The demographic data and patient characteristics in MICU and RICU are shown in Table 1. In MICU, a total of 110 prescriptions were analyzed during the study period which includes 77 male and 33 female patients. The average age of the patients was $48( \pm 16.8)$ years and average length of stay (LOS) was $4( \pm 2.5)$ days. A total of 1046 drugs were prescribed during the period of stay and AMAs accounted for $16 \%$ of total drugs.

In RICU, the prescriptions of 99 patients were analyzed. It included 58 males and 41 females. The average age of the study population was $54( \pm 21.7)$ years and the average LOS was $4( \pm 1.4)$ days. About, 1024 drugs were prescribed and AMAs constitute about $17 \%$ of the total drugs.

\section{AMA Frequently Prescribed}

Table 2 shows the most frequently prescribed AMAs in MICU and RICU with their DDD/100 bed days. In MICU, ceftriaxone usage was found to be more than $30 \%$ as compared to other AMAs. In RICU, almost 12 single AMAs were used in the treatment in patients. Among these, ceftriaxone was most frequently used AMA followed by clindamycin, while ceftazidime, cefotaxim and streptomycin were least prescribed.

\section{AMA Prescribed in Combination}

The most frequently prescribed AMAs in MICU and RICU as combination along with their DDD/100 bed days are summarized in Table 3. It was observed that piperacillin+ tazobactam combination $(26 \%)$ was found to be prescribed more in MICU, followed by cefoperazone+ tazobactam $(18 \%)$. 


\begin{tabular}{|c|c|c|c|c|c|}
\hline \multirow{2}{*}{\multicolumn{2}{|c|}{$\begin{array}{c}\text { Characteristics } \\
\text { Number }\end{array}$}} & \multicolumn{2}{|c|}{ RICU } & \multicolumn{2}{|c|}{ MICU } \\
\hline & & \multirow{2}{*}{$\frac{\text { Percentage (\%) }}{58}$} & \multirow{2}{*}{$\begin{array}{c}\text { Number } \\
62.7\end{array}$} & \multirow{2}{*}{$\frac{\text { Percentage (\%) }}{77}$} & \multirow{2}{*}{$\frac{\text { Number }}{70}$} \\
\hline \multirow{4}{*}{ Gender } & Male & & & & \\
\hline & Female & 41 & 37.3 & 33 & 30 \\
\hline & $15-30$ & 14 & 12.7 & 17 & 15.5 \\
\hline & $31-45$ & 20 & 18.1 & 30 & 27.2 \\
\hline \multirow{5}{*}{$\begin{array}{l}\text { Age distribution } \\
\text { (years) }\end{array}$} & $46-60$ & 21 & 19.1 & 37 & 33.6 \\
\hline & $61-75$ & 27 & 24.5 & 22 & 20 \\
\hline & $>75$ & 17 & 15.4 & 4 & 3.6 \\
\hline & Mean $\pm S D$ & \multicolumn{2}{|c|}{$54 \pm 21.7$} & \multicolumn{2}{|c|}{$48 \pm 16.8$} \\
\hline & $1-5$ & 13 & 11.8 & 15 & 13.6 \\
\hline \multirow{3}{*}{ Drugs prescribed } & $6-10$ & 39 & 35.4 & 53 & 48.1 \\
\hline & $\geq 11$ & 47 & 42.7 & 42 & 38.1 \\
\hline & Mean $\pm S D$ & \multicolumn{2}{|c|}{$10 \pm 5.0$} & \multicolumn{2}{|c|}{$10 \pm 4.8$} \\
\hline \multirow{4}{*}{ AMAs prescribed } & $1-2$ & 81 & 73.6 & 96 & 96.8 \\
\hline & $3-4$ & 16 & 14.5 & 12 & 3.2 \\
\hline & $\geq 5$ & 2 & 1.8 & 2 & 0 \\
\hline & Mean $\pm S D$ & \multicolumn{2}{|c|}{$2 \pm 0.9$} & \multicolumn{2}{|c|}{$2 \pm 1.0$} \\
\hline \multirow{6}{*}{ Length of stay } & $1-5$ & 86 & 78.1 & 89 & 80.9 \\
\hline & $6-10$ & 12 & 10.9 & 18 & 16.3 \\
\hline & $11-15$ & 0 & 0 & 3 & 2.7 \\
\hline & $>15$ & 1 & 0.9 & 0 & 0 \\
\hline & Mean $\pm S D$ & \multicolumn{2}{|c|}{$4 \pm 1.4$} & \multicolumn{2}{|c|}{$4 \pm 2.5$} \\
\hline & Medical & 85 & 77.3 & 72 & 65.5 \\
\hline \multirow[t]{2}{*}{ Department of origin } & Surgical & 13 & 11.8 & 18 & 16.3 \\
\hline & Trauma & 1 & 0.9 & 20 & 18.2 \\
\hline
\end{tabular}

\begin{tabular}{|c|c|c|c|c|c|c|c|c|c|c|}
\hline \multirow[b]{2}{*}{ Drug } & \multirow[b]{2}{*}{ ATC Code } & \multirow[b]{2}{*}{ Route } & \multicolumn{4}{|c|}{ MICU } & \multicolumn{4}{|c|}{ RICU } \\
\hline & & & $\begin{array}{l}\text { DDD } \\
\text { (g) }\end{array}$ & $\begin{array}{l}\text { Pres } \\
\text { (n) }\end{array}$ & $\begin{array}{c}\text { Total } \\
\text { Units }(g) \\
\end{array}$ & $\begin{array}{l}\text { DDD/100 } \\
\text { bed days }\end{array}$ & $\begin{array}{l}\text { DDD } \\
\text { (g) }\end{array}$ & Pres (n) & Total Units(g) & $\begin{array}{c}\text { DDD/100 bed } \\
\text { days }\end{array}$ \\
\hline Ceftriaxone & J01DD04 & $\mathrm{P}$ & 2 & 33 & 249 & 22.2 & 2 & 40 & 308 & 32 \\
\hline Clindamycin & J01FF01 & $\mathrm{P}$ & 1.8 & 12 & 60 & 5.9 & 1.8 & 17 & 111.6 & 12.9 \\
\hline Levofloxacin & J01MA12 & $\mathrm{O}$ & 0.5 & 1 & 1.5 & 0.5 & 0.5 & 5 & 5.5 & 2.3 \\
\hline Meropenam & J01DH02 & $\mathrm{P}$ & 2 & 8 & 37.5 & 3.3 & 0.5 & 7 & 11.5 & 4.8 \\
\hline Amikacin & J01GB06 & $\mathrm{P}$ & 1 & 17 & 38.7 & 6.9 & 2 & 11 & 62.5 & 6.5 \\
\hline Azithromycin & J01FA10 & $\mathrm{P}$ & 0.3 & 1 & 1 & 0.4 & 1 & 6 & 8.75 & 1.8 \\
\hline \multirow{2}{*}{ Ciprofloxacin } & \multirow{2}{*}{ J01MA02 } & $\mathrm{O}$ & 1 & 1 & 1 & 0.2 & 0.3 & 5 & 4.5 & 3.1 \\
\hline & & $\mathrm{P}$ & 0.5 & 1 & 1.5 & 0.5 & 1 & 3 & 2.5 & 0.5 \\
\hline Doxycycline & J01AA02 & $\mathrm{P}$ & 0.1 & 2 & 0.6 & 1 & 0.1 & 3 & 1.8 & 3.7 \\
\hline Vancomycin & J01XA01 & $\mathrm{O}$ & 2 & 9 & 48 & 4.3 & 2 & 3 & 27.5 & 2.9 \\
\hline Ceftazidime & J01DD02 & $\mathrm{P}$ & 4 & 2 & 16.4 & 0.7 & 4 & 1 & 1 & 0.05 \\
\hline Cefotaxim & J01DD01 & $\mathrm{P}$ & 4 & 2 & 27 & 1.2 & 4 & 1 & 6 & 0.3 \\
\hline Streptomycin & J01GA01 & $P$ & - & - & - & - & 1 & 1 & 1 & 0.5 \\
\hline Linezolid & J01XX08 & $\mathrm{P}$ & 1.2 & 1 & 3.6 & 0.5 & - & - & - & - \\
\hline Ampicillin & J01CA01 & $P$ & 2 & 1 & 8 & 0.7 & - & - & - & - \\
\hline Teicoplanin & J01XA02 & $P$ & 0.4 & 1 & 16 & 7.1 & - & - & - & - \\
\hline Colistin & J01XB01 & $\mathrm{P}$ & 3 & 3 & 14 & 0.8 & - & - & - & - \\
\hline
\end{tabular}

P-Parenteral, O-Oral, Pres - Prescription, DDD -Daily Defined Dose. 


\begin{tabular}{|c|c|c|c|c|c|c|c|c|c|c|}
\hline \multirow[b]{2}{*}{ Drug Combination } & \multirow[b]{2}{*}{ ATC Code } & \multirow[b]{2}{*}{ Route } & \multicolumn{4}{|c|}{ MICU } & \multicolumn{4}{|c|}{ RICU } \\
\hline & & & $\begin{array}{l}\text { DDD } \\
\text { (g) }\end{array}$ & $\begin{array}{c}\text { Pres } \\
\text { (n) }\end{array}$ & $\begin{array}{c}\text { Total } \\
\text { Units }(g)\end{array}$ & $\begin{array}{l}\text { DDD/100 } \\
\text { bed days }\end{array}$ & $\begin{array}{c}\text { DDD } \\
\text { (g) }\end{array}$ & $\begin{array}{c}\text { Pres } \\
\text { (n) }\end{array}$ & $\begin{array}{c}\text { Total } \\
\text { Units(g) }\end{array}$ & $\begin{array}{l}\text { DDD/100 } \\
\text { bed days }\end{array}$ \\
\hline Piperacillin+Tazobactam & J01CR05 & $\mathrm{P}$ & 14 & 29 & 708.7 & 9 & 14 & 39 & 1442.2 & 21.5 \\
\hline Cefuroxime+Sulbactam & J01RA03 & $\mathrm{P}$ & 3 & 10 & 156 & 9.3 & 3 & 3 & 31.5 & 2.1 \\
\hline Amoxcillin+Clavulanic acid & J01CR02 & $\mathrm{P}$ & 3 & 6 & 59.1 & 3.5 & 3 & 9 & 139.8 & 9.7 \\
\hline Cefoperazone+Tazobactam & J01DD62 & $\mathrm{P}$ & 4 & 20 & 189.9 & 8.5 & 4 & 6 & 54.7 & 2.8 \\
\hline Imipenam+Cilastatin & J01DH51 & $\mathrm{P}$ & 2 & 7 & 45.7 & 4 & 2 & 4 & 15 & 1.6 \\
\hline Cotrimoxazole+Sulfomethoxazole & J01EE01 & $\mathrm{O}$ & 1.9 & 4 & 115.2 & 10.7 & - & - & - & - \\
\hline Cotrimoxazole+Trimethoprim & J01EE01 & $\mathrm{O}$ & - & - & - & - & 1.9 & 5 & 134.4 & 14.6 \\
\hline Cefoperazone+Sulbactam & J01DD62 & $\mathrm{P}$ & 4 & 2 & 15 & 0.7 & 4 & 3 & 16.5 & 0.9 \\
\hline Meropenam+Sulbactam & J01DH50 & $\mathrm{P}$ & 2 & 1 & 22.5 & 2 & - & - & - & - \\
\hline Cefixime+Clavulanic acid & J01DD08 & $\mathrm{O}$ & - & - & - & - & 0.4 & 1 & 0.4 & 0.2 \\
\hline
\end{tabular}

P-Parenteral, O-Oral, Pres-Prescription, DDD-Daily Defined Dose.

\begin{tabular}{|c|c|c|c|c|}
\hline \multirow[b]{2}{*}{ Specimen } & \multicolumn{2}{|c|}{ MICU } & \multicolumn{2}{|c|}{ RICU } \\
\hline & Culture test done (n) & Growth present (n) & Culture test done (n) & Growth present (n) \\
\hline Blood & 44 & 13 & 40 & 7 \\
\hline Urine & 27 & 12 & 17 & 2 \\
\hline Sputum & - & - & 17 & 8 \\
\hline CSF & 6 & - & 3 & - \\
\hline Wound Swab & 3 & 4 & - & - \\
\hline Pus & 3 & 3 & - & - \\
\hline ETST & 5 & 3 & 7 & 2 \\
\hline ETS & 1 & 0 & 6 & - \\
\hline Ascitic fluid & 2 & 0 & 1 & - \\
\hline Pleural fluid & 1 & 0 & 1 & - \\
\hline Stool & 1 & 0 & 2 & - \\
\hline
\end{tabular}

CSF-Cerebrospinal Fluid, ETST-Endotracheal Suction Tip, ETS - Endotracheal Secretions.

Table 5: Frequency of microorganisms isolated from various specimens in MICU and RICU

\begin{tabular}{|c|c|c|c|c|c|c|c|c|c|c|c|c|c|c|}
\hline \multirow{3}{*}{ Microorganism } & \multicolumn{14}{|c|}{ Specimens } \\
\hline & \multicolumn{2}{|c|}{ Blood } & \multicolumn{2}{|c|}{ Urine } & \multicolumn{2}{|c|}{ Wound swab } & \multicolumn{2}{|c|}{ ETST } & \multicolumn{2}{|c|}{ Pus } & \multicolumn{2}{|c|}{ Sputum } & \multicolumn{2}{|c|}{ ETS } \\
\hline & M & $\mathbf{R}$ & M & $\mathbf{R}$ & M & $\mathbf{R}$ & M & $\mathbf{R}$ & $\mathbf{M}$ & $\mathbf{R}$ & M & $\mathbf{R}$ & M & $\mathbf{R}$ \\
\hline E. coli & 4 & 1 & 5 & 1 & 2 & - & 1 & - & 2 & - & - & - & - & 1 \\
\hline S. aureus & 2 & 1 & - & - & - & - & - & - & 1 & - & - & 1 & - & - \\
\hline Citrobacter sps & - & - & 2 & 1 & - & - & - & - & - & - & - & - & - & 1 \\
\hline Enterococcus sps & 1 & - & - & - & - & - & - & - & - & - & - & - & - & - \\
\hline K. pneumonia & 1 & - & 2 & - & 1 & - & - & 1 & - & - & - & 2 & - & - \\
\hline $\begin{array}{l}\text { Coagulase negative } \\
\text { staphylococci }\end{array}$ & 3 & 3 & - & - & - & - & - & - & - & - & - & - & - & - \\
\hline Enterobacter cloacae & 1 & - & - & - & - & & - & - & - & - & - & - & - & - \\
\hline Acinectobacter sps & 1 & 1 & 1 & - & 1 & - & 1 & 1 & 2 & - & - & 4 & - & - \\
\hline$P$. aeruginosa & - & - & 2 & - & - & - & 1 & - & - & - & - & 1 & - & 2 \\
\hline Gram positive cocci & - & 1 & - & - & - & - & - & - & - & - & - & - & - & - \\
\hline K. oytoxica & - & - & - & - & - & - & - & - & - & - & 1 & - & - & - \\
\hline
\end{tabular}

M-MICU, R-RICU, ETST-Endotracheal Suction Tip, ETS - Endotracheal Secretions. 
Benjamin et al.: Comparative Drug Utilization of Antimicrobial Agents in Medical and Intensive Care Units

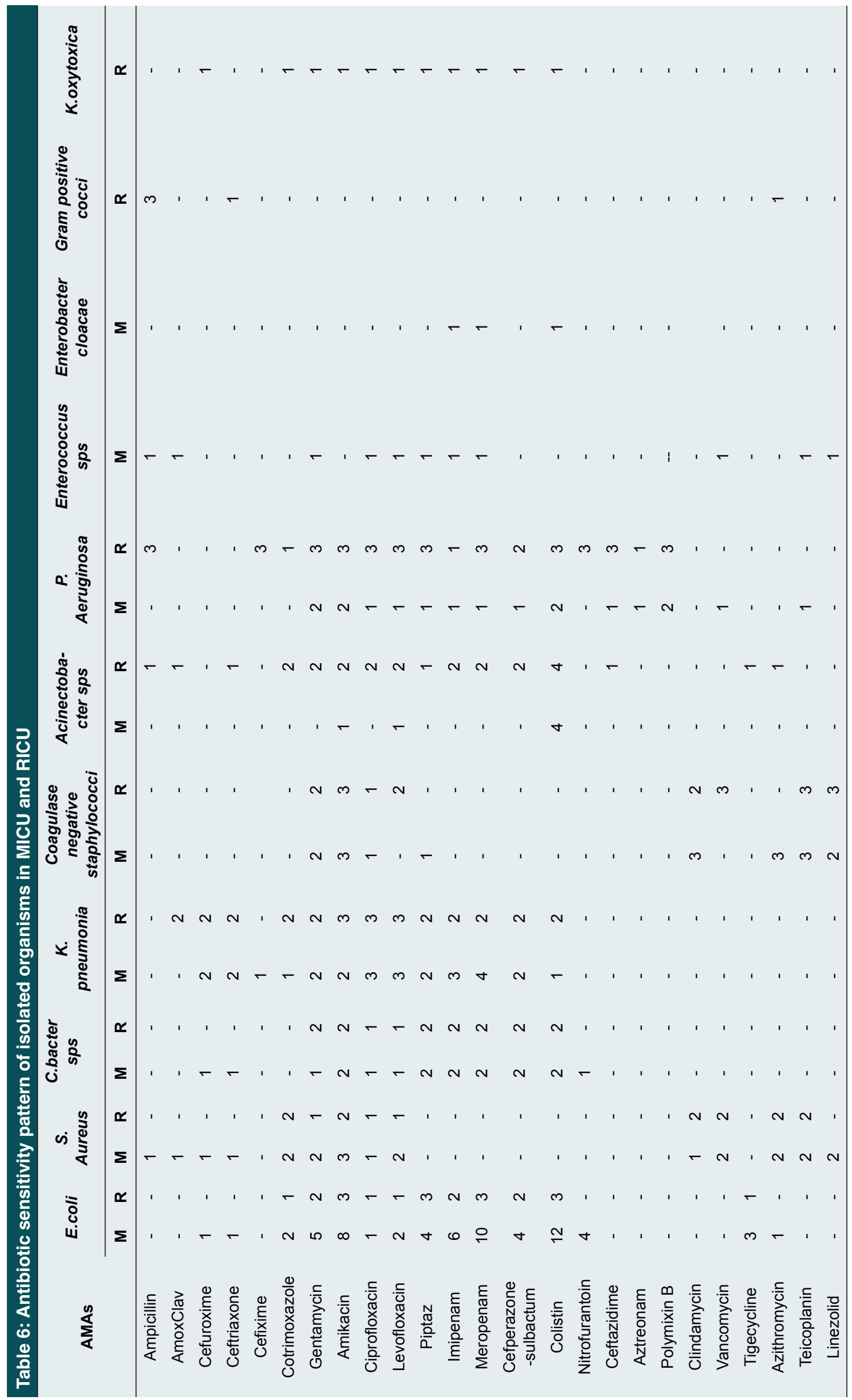

Indian Journal of Pharmacy Practice, Vol 9, Issue 2, Apr-Jun, 2016

127 
In RICU, Piperacillin+tazobactam (39\%) was the most frequently used AMA combination followed by amoxicillin+clavulanic acid (9\%).

\section{Class and Types of AMA prescribed}

In MICU and RICU, most of the patients were prescribed with 1 and 2 AMAs. In MICU, the percentage of AMA prescribed for 1 and 2 AMAs were $53 \%$ and $35 \%$, while in RICU it was $40.4 \%$ and $39 \%$ respectively. Figure 1 shows the class of AMA prescribed in MICU and RICU. The distributions of patients according to number of AMAs prescribed are illustrated in Figure 2.

The types of dosage forms (AMAs) prescribed were also monitored. In MICU, injections (98\%) were widely prescribed followed by tablets $(10 \%)$. In RICU, $95 \%$ of the prescriptions contain AMA as injections followed by tablets $(17 \%)$. Other dosage forms prescribed in MICU and RICU include ointments and drops, followed by capsules, inhalations and creams (all below 5\%).

\section{Culture Pattern for Various Specimens}

The culture patterns in MICU and RICU for various specimens are shown in Table 4. In MICU, out of 52 (47\%) samples screened for microbial sensitivity, 26 (50\%) samples exhibited microbial growth. The chief organisms revealed in blood culture report in MICU were E.coli (53\%) and Acinetobacter sps (23\%). In RICU, out of 99 patients, $54(55 \%)$ patients were subjected to culture test. It was observed that $20(37 \%)$ patient's sample contain pathogenic microorganisms. Acinetobacter sps (25\%) was the most frequently isolated bacteria, followed by E.coli, Coagulase negative staphylococci and Klebsiella pneumonia, each constituting around $15 \%$.

\section{Frequency of Microorganism and Antibiotic Culture Sensitivity Pattern}

The frequency of microorganisms isolated from various specimens and antibiotic sensitivity pattern of isolated organisms in MICU and RICU are shown in Table 5 and Table 6 respectively.

\section{DISCUSSION}

DUE is an inexpensive, flexible and simple method to assess the utilization pattern of drug. DUE has defined potential benefits both for patient and for clinical practice in carrying out a well planned audit of prescribing drug. ${ }^{7}$ The present study was performed to evaluate and improve the use of antimicrobials in critical care patients. During the 6 month study period, we audited 209 prescriptions with antimicrobials from various intensive care units (MICU-110, RICU-99). Culture sensitivity reports were obtained from hospital laboratory. The cost incurred for various AMAs were also noted.
The data collected were analyzed and summarized accordingly.

Medical ICU (MICU) is a unit providing acute care for adult and geriatric critically ill patients. The demographic results of patients revealed that more male patients were admitted in Medical ICU. The mean age of patients was $48( \pm 16.8)$ years and the average LOS was $4( \pm 1.4)$ days. The data was similar to studies conducted by Shankar PR et al., (2003), in Intensive Care Unit of a teaching hospital in Western Nepal. ${ }^{8}$

In MICU, a mean of $10( \pm 4.1)$ drugs and a mean of $2( \pm 1.0)$ AMAs were prescribed per patient. The study shows that the prescription of AMAs in MICU was less in comparison to another hospital in North India $(3.36 \%) .{ }^{9}$ Similarly, studies from Danish university ICU hospital shows many of their patients were prescribed with only one AMA. ${ }^{10}$ In our observation most of the patients received one or two AMAs.

In ICU, the most frequently prescribed AMAs were ceftriaxone followed by amikacin and clindamycin. The AMAs in MICU were found to vary in comparison to other study in India (Data not reported). In the later case, the most commonly prescribed AMAs were cefoperazone, amikacin and metronidazole. ${ }^{11}$ The DDD/100 bed days for ceftriaxone, amikacin and clindamycin were found to be 22.2, 6.9 and 5.9 respectively. The generally prescribed AMA combination was piperacillin+tazobactam and cefoperazone + tazobactam. The laboratory reported positive cultures for 26 patients. The most prevailing organisms were E.Coli (53\%) and Acinetobacter sps (23\%). Colistin and meropenam were the most sensitive AMA for most microorganisms.

Respiratory ICUs (RICU) are specialized units for monitoring and treatment of acute or chronic respiratory failure by non invasive and invasive ventilation. Prescriptions of 99 patients admitted to RICU were audited to study the drug utilization pattern. Male to female ratio was $1: 4$ and average LOS was $4( \pm 1.4)$ days. Results indicated that the patients of 61-75 years received more AMAs than any other age group. An average of $10( \pm 5.0)$ drugs were prescribed for each patient and each prescription contains an average of $2( \pm 0.9)$ AMAs.

A total of 12 antimicrobial monotherapy were prescribed for RICU patients. The results indicated that ceftriaxone was the most commonly prescribed AMAs $(40 \%)$ followed by clindamycin (17\%), levofloxacin $(12 \%)$ and meropenam $(11 \%)$. A total of 8 AMA combination therapies were used in RICU. Among them, piperacillin+tazobactam (30\%) was the most commonly prescribed combination. The DDD/100 bed days were also high for the above AMAs. Out of 99 cases prescribed with AMAs, only in $54(54 \%)$ cases blood 


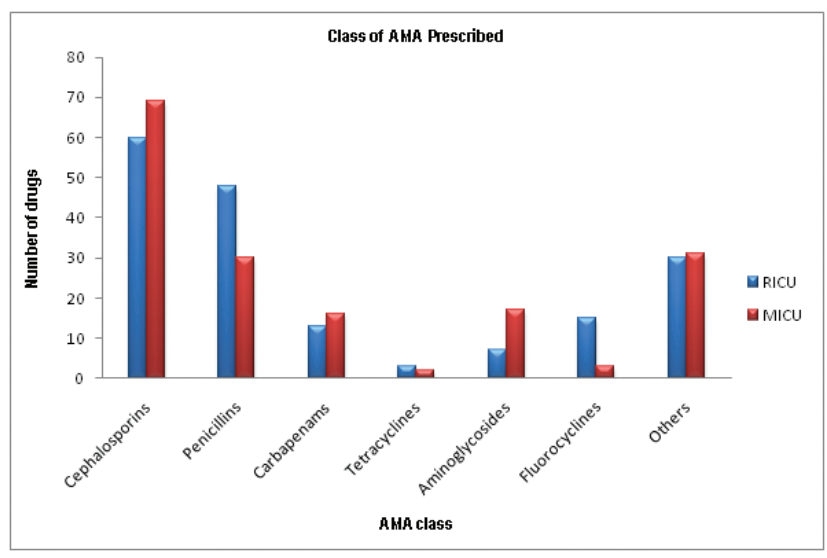

Figure 1: Comparison of AMA class prescribed in RICU and MICU.

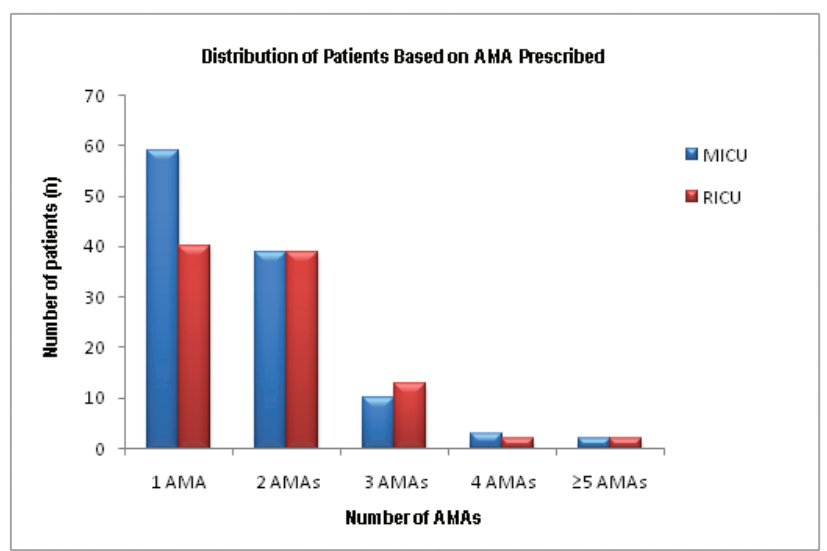

Figure 2: Distribution of patients according to number of AMAs prescribed in MICU and RICU.

culture test was performed. In our observation, 20 (37\%) cases had microbial growth and have performed sensitivity test. Most of the microbial growth was found in sputum. Acinetobacter sps was the most frequently isolated pathogen and almost all microorganisms were found to be sensitive to colistin.

The prescription of AMAs in the hospital setting is often empiric, especially in the critically ill patients. In our study site, the total number of beds in MICU and RICU were 10 each and the occupancy indices of MICU and RICU were 0.7 and 0.6 respectively. The demographic results of the patients admitted to MICU and RICU over a period of 3 months revealed male preponderance. The probable reason for this finding could be the higher male to female ratio in the state of Karnataka ${ }^{12} \mathrm{Also}$, in the Indian set-up it is noticed that female population were unwilling to make use of healthcare services even if they are critically ill. ${ }^{13}$ The average
LOS (4 days) and the mean total number of drugs prescribed (10 Nos) for critically ill patients were comparable in both ICUs. The average numbers of AMAs per patient in both ICU were 2 . In our examination in both ICUs, mostly medical patients were admitted, and more surgical and traumatic patients were admitted in MICU.

In MICU, 15 AMA monotherapies and 9 AMA combination therapies were prescribed, while in RICU, around 12 AMA monotherapies and 7 AMA combination therapies were given to the patients. The extensively prescribed monotherapy in both ICUs was ceftriaxone. The probable reason could be due to its extreme long half-life $(8 \mathrm{~h})$.Use of streptomycin was observed only in RICU, while in MICU it was not prescribed at all. Piperacillin+tazobactam combination was mostly prescribed AMA in critically ill patients. Meropenam+sulbactam and cefadroxyl+clavulanate combination were only prescribed in MICU patients. With respect to the type of drug dosage form, injections were mostly prescribed in both the ICUs.

An appropriate method of treatment involves selection and utilization of AMA by considering the sensitivity pattern of antibiotics. ${ }^{14}$ The sensitivity pattern studies can reflect the major organisms to be treated and could help to choose the suitable AMAs for the proper treatment. In our observation, culture sensitivity test was done in 52 patients in MICU whereas, the test was performed on 54 patients in RICU. Microbial growth was found in 26 samples from MICU and 20 samples showed the growth from RICU. In both ICUs, blood was the major specimen in which culture test was done. Microbial growth was found to be more in blood (MICU) and in sputum (RICU). E.coli was the most commonly isolated microorganism in MICU whereas, Acinetobacter species was the most prevailing organism in RICU patients. In our observation, E.Coli was highly sensitive to colistin, meropenam and amikacin, while Acinetobacter sps was more sensitive to colistin. It was also observed that cephalosporins were highly resistant to microorganism. The high degree of resistance to cephalosporins may be probably due to the extensive use of the drugs in the hospital.

The study reveals that there is a high rate of consumption of AMAs in the ICUs. Such inappropriate use of AMAs can result in increasing antibiotic resistance. Therefore, health system policy makers should consider implementing reasonable administration guidelines for AMAs through appropriate drug policies. ${ }^{15}$ In future, pharmacists have a greater responsibility to take prominent roles in antimicrobial stewardship and infection prevention and control programs in healthcare systems. 


\section{CONCLUSION}

A comparative drug utilization study of AMA was conducted in tertiary level teaching hospital in South India. The study reveals that a wide class and percentage of AMAs were prescribed in ICUs. Majority of patients received more than two AMAs during the ICU stay. As the patients were severely ill, majority of the AMAs were prescribed as injections. Ceftriaxone was the most frequently prescribed monotherapy and piperacillin+ tazobactam was the combinative drug used in critically ill patients. The study reveals that appropriate use of AMAs and culture sensitivity pattern in ICUs is highly beneficial for the proper functioning of the hospital.

Study on organisms prevailing and its sensitivity pattern of AMAs in the ICU will help the physicians to select the proper drug of choice. Even though high degrees of resistance to cephalosporins were observed in ICUs, it was mostly prescribed in critically ill patients. For proper AMA prescribing, the clinician may consider initiating the therapy based on antibiogram. Also, the dose adjustment in patient may be done based on further results of culture sensitivity tests. Clinical pharmacists and physicians must play a crucial role in minimizing the problems associated with irrational use of antimicrobial agents by conducting awareness programs among other healthcare professionals. The development and regular updation of antimicrobial use guidelines and restriction policies could be useful in promoting better patient care.

\section{ACKNOWLEDGEMENTS}

The authors would like to thank Rev. Fr Patrick Rodrigues, Director, Father Muller Medical College Hospital (FMMCH) and Charitable Institutions, Mangalore for granting us necessary permission for the study. The authors also thank all the healthcare professionals of Intensive Care Units of FMMCH for their constant support and guidance.

\section{CONFLICT OF INTEREST}

The authors declare no conflict of interest.

\section{ABBREVIATION USED}

MICU: Medical Intensive Care Unit; RICU: Respiratory Intensive Care Unit; DDD: Defined Daily Dose; LOS: Length of Stay; AMA: Antimicrobial Agent.

\section{REFERENCES}

1. Introduction to Drug Utilization Research, WHO, 2013. Available at http://www. whocc.no/filearchive/publications/drug_utilization_research.pdf. Accessed 07 August 2015.

2. How to Investigate Antimicrobial Use in Hospitals: Selected Indicators. Available at http://apps.who.int/medicinedocs/en/d/Js21031en.Accessed 10 June 2015.

3. Charles-Edouard Luyt, Nicolas Brechot, Jean-Louis Troullit, Jean Chastre. Antibiotic stewardship in the intensive care unit. Critical Care. 2014;18(5):480.

4. Andrew Berrington. Antimicrobial prescribing in hospitals: be careful what you measure. Journal of Antimicrobial Chemotherapy. 2010;65(1)163-8.

5. Ahmed Al Ansari, Mohammed Azizullah Khan, Jalal Khan, Mohammed Lamine Hariz. Antibiotic Prescription Patterns in an Intensive care unit in the Kingdom of Bahrain : An Observational Prospective Study. International Journal of Scientific Research. 2013;2(12)371-4.

6. Williams A, Mathai AS, Philips AS. Antibiotic prescription patterns at admission into tertiary level intensive care unit in Northern India. J Pharm Bioallied Sci 2011;3(4)531-6.

7. A study on drug utilization pattern of antimicrobials in outpatient department of medicine at tertiary care hospital. Int J Res Pharm Sci. 2014;4(2)40-5.

8. Shankar PR, Partha $P$, Shenoy $N$, Brahadathan $K N$. Investigation of antimicrobial use pattern in the intensive care unit of a teaching hospital in Western Nepal. Am J Infect Control. 2003; 31(7)410-4.

9. Bharti Mahajan, Sandeep Kaushal, Sarvesh Chander Chopra. A drug utilization study of Antimicrobial agents (AMAs) in the intensive care units (ICUs) at Medical College Hospital of North India. JK Science. 2013;15(3)129-32.

10. Hartmann B, Junger A, Brammen D, Röhrig R, Klasen J, Quinzio L, et al. Review of antibiotic drug use in a surgical ICU: management with a patient data management system for additional outcome analysis in patients staying more than 24 hours. Clin Ther. 2004;26(6)915-24.

11. Rutvij Hedamba, Chintan Doshi, Nishita H. Darji, Bansari Patel, Varsha Kumari. Drug utilization pattern of antimicrobial drugs in intensive care unit of a tertiary care hospital attached with a medical college. International Journal of Basic \& Clinical Pharmacology.| 2016;5(1)169-72.

12. Lisha Jenny John, Padmini Devi, Jenny John, Shoba Guido. Drug Utilization Study Of Antimicrobial Agents In Medical Intensive Care Unit Of A Tertiary Care Hospital. Asian Journal of Pharmaceutical and Clinical Research. 2011;4(2)81-4.

13. Ministry of Home Affairs. Directorate of Census Operations Karnataka Census - 2011 http://censuskarnataka.gov.in/. Accessed 23 June 2016.

14. Omid Adeli, Nader Markazi Moghaddam, RaminHamidi Farahani, Sanaz Zargar. Antibiotics Use Patterns in Intensive Care Units of Five Hospitals in Tehran During 2011-2012. J Arch Mil Med. 2015;3(3)1-4.15.

15. ASHP Statement on the Pharmacist's role in Antimicrobial Stewardship and Infection Prevention and Control. Am J Health-Syst Pharm. 2010;(1)67:575-7. 